\title{
Interactions of High Energy Cosmic Rays with Extragalactic Infrared Radiation Background
}

\author{
Edgar Bugaev ${ }^{1, *}$ and Peter Klimai ${ }^{1, * *}$ \\ 1 Institute for Nuclear Research, Russian Academy of Sciences, \\ 60th October Anniversary Prospect 7a, 117312 Moscow, Russia
}

\begin{abstract}
We consider the modification of extragalactic cosmic ray spectrum caused by cosmic ray interactions with infrared background photons which are present in the extragalactic space together with relic photons. It is assumed that cosmic ray spectrum at superhigh energies has extragalactic origin and is proton dominated.
\end{abstract}

\section{INTRODUCTION}

Presence of cosmic infrared background radiation (CIB) in extragalactic space has now been confirmed by experiments, and its intensity is measured (see, e.g., reviews [1, 2]), though the precision of these measurements is still not so high, especially if to compare with those for relic radiation. Nevertheless, we know enough to estimate some effects caused by the presence of the extragalactic infrared background. One of such effects is neutrino production due to high energy cosmic ray proton interactions with CIB photons, considered in [3-5]. Here we will focus on the other point: high energy cosmic ray spectrum modification due to interactions with CIB photons.

\section{EXTRAGALACTIC COSMIC RAY PROTON SPECTRUM}

For the calculation of cosmic ray $(\mathrm{CR})$ energy spectrum at high energies, we assume it to be extragalactic and proton dominated from the energy $E_{0}=3 \times 10^{17} \mathrm{eV}$. The sources of these CRs are assumed to be distributed isotropically and uniformly throughout the Universe. In

\footnotetext{
* Electronic address: bugaev@pcbai10.inr.ruhep.ru

** Electronic address: pk@pochta.ru
} 
this case it is very convenient to use cosmological transport equation written as

$$
\frac{\partial n(E, z)}{\partial z}+\frac{\partial}{\partial E}[\beta(E, z) n(E, z)]-\frac{3 n(E, z)}{1+z}=g(E, z) .
$$

We work in in the continuous energy loss approximation introduced in [6] and neglect possible proton absorbtion. In (1), $n(E, z)$ is the number density of CR protons with a given redshift $z$, the function $\beta(E, z)$ is the change of proton energy in unit interval of $z$,

$$
\beta(E, z)=\frac{d E}{d z}=\frac{E}{1+z}-b(E, z) \frac{d t}{d z}
$$

where the first term in r.h.s. is due to adiabatic energy losses (caused by cosmological expansion $), b(E, z)=-d E / d t$, and is simply connected with the proton cooling rate $t_{p}^{-1}(E, z)$. For the case of proton interactions with photon gas it is given by the formula

$$
t_{p}^{-1}(E, z)=\frac{1}{E} b(E, z)=\frac{c}{2 \gamma_{p}^{2}} \int_{\epsilon_{t h}}^{\infty} d \epsilon_{r} \sigma\left(\epsilon_{r}\right) f\left(\epsilon_{r}\right) \epsilon_{r} \int_{\epsilon_{t h} / 2 \gamma_{p}}^{\infty} d \epsilon \frac{n^{\text {photon }}(\epsilon, z)}{\epsilon^{2}}
$$

where $\gamma_{p}=E / m_{p}$ is proton Lorenz factor, $\epsilon_{r}$ is the photon energy in the CR proton rest system, $\sigma\left(\epsilon_{r}\right)$ is the photoabsorbtion cross section, $f\left(\epsilon_{r}\right)$ is the average relative proton energy loss in $p \gamma$-collision (in the observer system), $\epsilon_{t h}$ is the threshold of the photoabsorbtion reaction.

Protons lose their energy interacting with photons via $p \gamma \rightarrow \pi X$ and $p \gamma \rightarrow p e^{+} e^{-}$reactions. Here, we suppose that $\gamma$ can be relic photon (they have black body spectral distribution with $T \approx 2.7 \mathrm{~K}$ ) or background infrared photon.

Thus, in our approximation the total cooling rate should be written as a sum of two components: cooling due to interactions with relic and infrared components of the extragalactic radiation background,

$$
t_{p}^{-1}(E, z)=t_{p, r e l i c}^{-1}(E, z)+t_{p, \text { infr }}^{-1}(E, z)
$$

The function $g(E, z)$ in r.h.s. of the kinetic equation (1) describes the combined source of extragalactic cosmic rays. This source function can be written in the form

$$
g(E, z)=\rho(z) \eta(z) f(E) \frac{d t}{d z} .
$$

Here, $\rho(z)$ is the number density of local CR sources (e.g., AGNs) in the proper (physical) volume, $\rho(z)=\rho_{0}(1+z)^{3}, \eta(z)$ is the activity of each local source (the integrated number of produced particles per second $), \eta(z)=(1+z)^{m} \eta_{0} \theta\left(z_{\max }-z\right)$. Writing this, we assume that 
the cosmological evolution of cosmic ray sources can be parametrized by power law with the sharp cut-off at some epoch with redshift $z_{\max }\left(m\right.$ and $z_{\max }$ are considered as parameters of a model of the combined source). At last, the function $f(E)$ in eq.(2) describes a form of the differential energy spectrum of the local source. For a simple power law injection spectrum we can write

$$
f(E)=\frac{\gamma-1}{E_{0}}\left(\frac{E}{E_{0}}\right)^{-\gamma}
$$

Now supposing some values for the presented parameters we can solve (1). One more thing needed is infrared photon number density for different redshifts, $n^{I R}\left(E_{\gamma}, z\right)$, to calculate energy losses in infrared background. As for the case of relic photons, the dependence of $n^{\text {relic }}\left(E_{\gamma}, z\right)$ on $z$ is trivial: they always have black body spectrum, with temperature $T(z)=T(0)(1+z)$.

\section{COSMIC INFRARED RADIATION BACKGROUND}

Extragalactic infrared background had been formed by infrared-luminous galaxies in the late Universe. For the calculation of CIB it is again convenient to use the cosmological transport equation which is analogous to that used in the previous section. The function which must be found is the number density of infrared photons at different cosmological epochs, $n^{I R}\left(E_{\gamma}, z\right)$.

The resulting expression for the number density of infrared photons in extragalactic space is $[4]$

$$
n^{I R}\left(E_{\gamma}, z\right)=\int_{z}^{z_{\max }} d x\left(\frac{1+z}{1+x}\right)^{3} \int \frac{d L}{L} \rho(x, L) S^{I R}\left(E_{\gamma} \frac{1+x}{1+z}, L\right) \cdot \frac{1}{E_{\gamma}}\left|\frac{d t}{d x}\right|,
$$

where $L$ is the luminosity of infrared-bright galaxy emitting photons, $S^{I R}\left(E_{\gamma}, L\right)$ describes the spectrum of this radiation (it is called the spectral energy distribution), $\rho(z, L)$ is the number density of infrared-bright galaxies with a given luminosity.

From direct measurements we can learn $\rho(0, L)$ and $S^{I R}\left(E_{\gamma}, L\right)$. Then we can assume some kind of the cosmological evolution to obtain $\rho(z, L)$. Next step is to calculate $n^{I R}\left(E_{\gamma}, 0\right)$ making use of formula (3), and compare it with the measured CIB intensity. One of the results of such estimations is the well-known fact $[1,2]$ that we need to assume strong evolution of background sources in the far infrared region to explain observed CIB intensity (say, $\nu I(\nu) \sim 30 n W m^{-2} s r^{-1}$ at $\lambda \sim 100 \mu m$ ). 
In fact, we include in our calculations only far and partially middle infrared background regions, not considering near infrared and optic regions. For the present calculations, we used the same parametrization of the function $\rho(z, L)$ and same input data as described in [5]. But we should mention that the effect under discussion (CR spectrum modification) does not depend greatly on the type of infrared sources evolution, but much more on the CIB intensity at $z=0$ (that is, at our time).

\section{RESULTS AND CONCLUSIONS}

On Fig. 1a we show the proton cooling rate in infrared photon gas (together with corresponding function for the relic photon case), both curves shown for $z=0$. The asymptotic value of $t_{p}^{-1}$ at highest energies is proportional to the total number density of photons, so for $E>10^{20} \mathrm{eV}$ cooling on infrared photons gives a small contribution, $\sim 0.5 \%$. But one can see from the figure that at proton energy $\sim(3 \div 4) \times 10^{19} \mathrm{eV}$, the contribution of infrared photons in total cooling rate is noticeable.

For calculation of the CR spectrum we used the following set of parameters determining the spectrum slope and cosmological evolution of CR sources: $\gamma=2.5, m=3.5, z_{\max }=5$. We assumed here that extragalactic spectrum of CRs dominates beginning from $E_{0}=3 \times$ $10^{17} \mathrm{eV}$. The theoretical CR spectrum at $z=0$ was normalized on experimental data; from such a normalization we obtained the value of the product $\rho_{0} \eta_{0}$ entering eq.(2) for the source function of the $\mathrm{CR}$ proton kinetic equation, $\rho_{0} \eta_{0} \approx 1.4 \times 10^{-42} \mathrm{~cm}^{-3} \mathrm{~s}^{-1}$.

The results of two calculations of extragalactic CR proton spectrum (with and without taking into account energy losses on infrared photons) are shown on Fig. 1b. Both curves have characteristic dip and cut-off features; one can see that extragalactic CR spectrum near GZK cut-off gets modified when taking into account interactions with infrared background.

In this work we used concrete model parameters for CR source properties and the certain model for CIB, for the estimation of CR extragalactic spectrum modification at $10^{19}-10^{20} \mathrm{eV}$. Certainly, new experimental data for CIB intensity as long as improvement of our knowledge 
a)

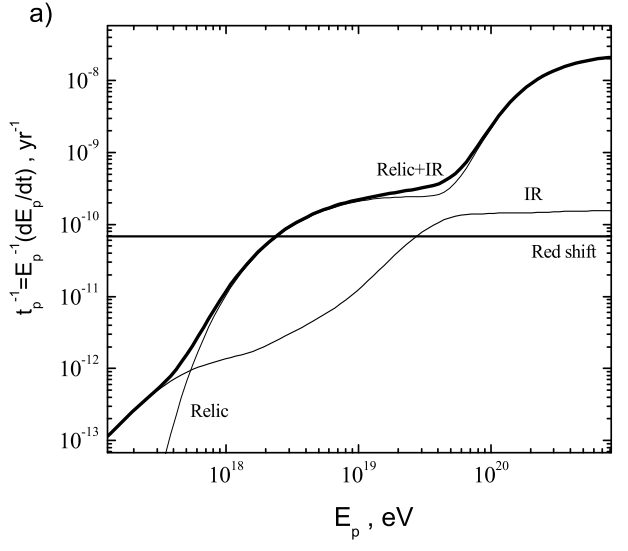

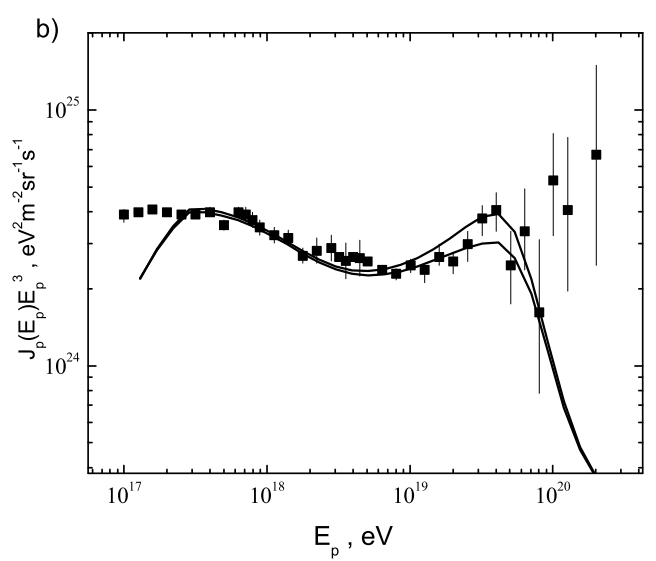

Figure 1. a) Proton cooling rates in relic and infrared photon background. b) Extragalactic $\mathrm{CR}$ proton spectrum calculated with (lower curve) and without (upper curve) taking into account energy losses in the infrared background.

of CR sources will help to determine more precisely the magnitude of this effect.

1. \refitem $\{$ article $\}$

Franceschini A., astro-ph/0009121.

2. \refitem $\{$ article $\}$

Hauser M. G. and Dwek, E., astro-ph/0105539.

3. \refitem $\{$ article $\}$

Stanev, T., Phys. Lett. B 595, 50, 2004.

4. \refitem $\{$ article $\}$

Bugaev, E. V., Misaki, A., and Mitsui, K., astro-ph/0405109.

5. \refitem $\{$ article $\}$

Bugaev, E.V, Klimai, P.A., astro-ph/0507366

6. \refitem $\{$ article $\}$

Berezinsky, V. S. and Grigorieva, S. I. 1988, A\&A, 199, 1. 